[gw22-e1045]

\title{
ASSOCIATION OF ALOX5AP GENE SG13S114T/A POLYMORPHISM WITH ACUTE CORONARY SYNDROME
}

Guoping HE, Jingjiao HUI, Dandan SHEN Department of Cardiology, Affiliated Wujin Hospital of Jiangsu University, Changzhou, China

\subsection{6/heartjnl-2011-300867.115}

Objective To investigate the distribution of ALOX5AP gene SG13S114T/A polymorphism and the association of the ALOX5AP gene SG13S114T/A polymorphism with acute coronary syndrome (ACS) in the Chinese Han population of Sunan region.

Methods This study was conducted with a case-control design including 545 patients with ACS (ACS group) and 567 control subjects who were free from coronary artery disease (control group). ALOX5AP gene SG13S114T/A polymorphism was determined by polymerase chain reaction and restriction fragment length polymorphism analysis.

Results There were AA, AT and TT genotypes of the ALOX5AP gene SG13S114T/A polymorphism both in ACS group and control group. The genotype distribution of the ACS group and control group conformed to the Hardy-Weinberg balance via $\chi^{2}$ test ( $p>0.05$ ), which suggested that the selected sample was representative. As compared with those in the control group, the genotype frequency of AT ( $37.57 \%$ vs $48.99 \%$, p $=0.015)$ was higher, the genotype frequency of TT $(48.15 \%$ vs $38.17 \%$, $\mathrm{p}=0.034)$ was lower, and the frequencies of AA genotype $(14.28 \%$ vs $12.84 \%, \mathrm{p}=0.054)$ and $\mathrm{T}$ allele $(66.93 \%$ vs $62.66 \%$, $\mathrm{p}=0.330$ ) were not significantly different (all $\mathrm{p}>0.05$ ) in ACS group. Subgroup analysis showed that as compared with those in control group respectively: (1) the genotype frequency of AT $(30.30 \%$ vs $56.41 \%, p=0.003)$ was higher, the genotype frequency of TT $(53.30 \%$ vs $32.50 \%, p=0.017)$ was lower, and the frequencies of AA genotype (16.67\% vs $13.54 \%, p=0.263$ ) and $\mathrm{T}$ allele $(68.33 \%$ vs $60.26 \%, \mathrm{p}=0.331)$ were not significantly different (all $p>0.05$ ) in AMI group; (2) the genotype frequency of AT ( $30.30 \%$ vs $47.14 \%, p=0.045)$ was higher, the frequencies of AA $(16.67 \%$ vs $10.00 \%, p=0.146)$ and TT $(53.33 \%$ vs $42.86 \%, p=0.291)$ genotypes and $T$ allele $(68.33 \%$ vs $60.26 \%$, $\mathrm{p}=0.727$ ) were not significantly different (all $\mathrm{p}>0.05)$ in UAP group; (3) the frequency of $\mathrm{T}$ allele $(68.06 \%$ vs $82.42 \%$, $\mathrm{p}=0.046)$ was higher in male ACS group and not significantly different $(p>0.05)$ in female ACS group, there were no significant statistical difference of the genotype frequencies of $\mathrm{AA}$ $((13.19 \%$ vs $8.02 \%)$ and $(5.41 \%$ vs $14.04 \%))$, AT ((37.50\% vs $33.69 \%)$ and $(37.99 \%$ vs $45.03 \%))$ and TT ((49.31\% vs $58.29 \%)$ and $(46.59 \%$ vs $40.94 \%))$ in male ACS group and female ACS group (all $p$ value $>0.05$ ); (4) the genotype frequency of AT $(38.58 \%$ vs $50.93 \%, p=0.041)$ was higher, the genotype frequency of TT $(48.66 \%$ vs $35.28 \%, p=0.020)$ was lower, the frequencies of AA ( $12.76 \%$ vs $13.79 \%, p=0.722)$ genotype and T $(61.22 \%$ vs $60.74 \%, p=0.931)$ allele were not significantly different in elderly ACS group; (5) the frequencies of AA (16.96\% vs $10.11 \%, \mathrm{p}=0.127)$, AT (36.09\% vs $50.00 \%, \mathrm{p}=0.078)$ and TT $(46.96 \%$ vs $39.39 \%$, $(p=0.338)$ genotypes and $\mathrm{T}$ allele $(65.00 \%$ vs $64.29 \%, p=0.932$ ) were not significantly different in premature ACS group. Multivariate logistic regression analysis showed that there was statistically significant correlation of AT and TT genotype, and T allele with ACS (P was 0.001, 0.001 and 0.031 , respectively). Furthermore, subgroups analysis showed that AT and TT genotype were correlated with AMI (all p<0.001); AT genotype was correlated with UAP $(p=0.007) ;$ AT and TT genotypes, and T allele were correlated 


\section{Abstracts}

with male ACS ( $p<0.001$, was 0.001 and 0.016 , respectively); AT and TT genotypes, and T allele were correlated with the elderly ACS (p was 0.004, 0.001 and 0.013, respectively).

Conclusion Three genotypes including AA, AT and TT genotypes exist in the ALOX5AP gene SG13S114T/A both in ACS group including its subgroups and control group. There was statistically significant association of the SG13S114T/A polymorphism of ALOX5AP gene with risk of ACS, AMI, UAP, male ACS and the elderly ACS in the Chinese Han population of Sunan region, which suggest ALOX5AP gene SG13S114T/A polymorphism play a potential role in the origin and development of ACS. 